\title{
Lipid profile and lipoprotein(a) in chronic renal failure patients with and without hemodialysis
}

\author{
Hariom Sharma', Tejas J. Shah², Jignesh H. Gorasia ${ }^{3}$ and Dipika P. Baria² \\ ${ }^{1}$ Govt. Medical College, Bhavnagar, Gujarat, India \\ ${ }^{2}$ M.P.Shah Medical College, Jamnagar, Gujarat, India \\ ${ }^{3}$ P.D.U. Medical College, Rajkot, Gujarat, India
}

Submission Date: 5-7-2012; Review Completed: 28-7-2012; Accepted Date: 2-8-2012

\section{A BSTRACT}

Objectives: Chronic renal failure (CRF) is complicated by characteristic dyslipidemias. CRF patients on hemodialysis have abnormalities in lipid profile and have a high incidence of cardiovascular diseases. Lipoprotein(a) [Lp(a)] is now considered as a novel cardiovascular risk factor and its level is increased in CRF patients with and without hemodialysis. We sought to evaluate the pattern of lipid profile including Lp(a) level in CRF patients with and without hemodialysis. Methodology: Study were divided into 3 groups, Group-I: healthy controls (30), Group-II: CRF patients who never undergone hemodialysis (30) and Group-III: CRF patients on hemodialysis for more than 6 months (30). We obtained serum samples from patients in the morning after an overnight fast and were analysed for total cholesterol (TC), triglycerides (TGs), HDL, LDL, Lp(a) using standard colorimetric assays on fully automated analyzer. VLDL concentration was calculated using Friedewald's Formula. Results: Among the various parameters tested triglyceride and VLDL levels were significantly higher in group-II and III as compared to controls $(p<0.01)$. HDL levels were significantly lower in group-II and III as compared to control $(p<0.05)$. HDL level was found reduced in group-III as compared to Group-II $(p<0.01)$. There was no significant change $(p>0.05)$ observed in total cholesterol and LDL levels in between healthy controls and CRF patients with \& without hemodialysis. Lp(a) levels were significantly higher in group-II and III as compared to controls $(p<0.01)$ but the difference between $L p(a)$ levels in group-II and III was found insignificant $(p>0.05)$. There was no significant difference $(p>0.05)$ observed between Lp(a) levels and lipid profile in male and female patients in control group and in CRF patients with and without hemodialysis. Conclusions: This study demonstrated that CRF patients with and without hemodialysis are at greater risk of development of dyslipidemias, characterized by hypertriglyceridemia, elevated VLDL and Lp(a) levels and decreased HDL levels. Total cholesterol and LDL cholesterol levels remain normal or decreased in these patients. Both male and female patients of CRF with and without hemodialysis have dyslipidemias without any discrimination of sex and it is not attenuated by the hemodialysis process.

Keywords: Chronic renal failure, Lipid profile, Lipoprotein(a), Hemodialysis

\section{INTRODUCTION}

Chronic renal failure (CRF) is a syndrome of persistent renal impairment involving loss of both glomerular and tubular function. ${ }^{1}$ Progressive CRF not only leads to end

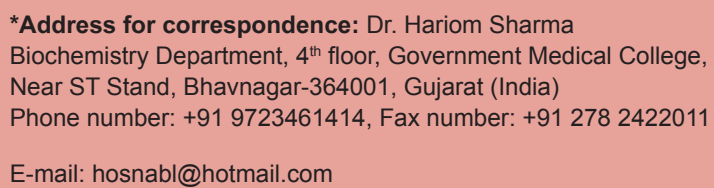

DOI: $10.5530 /$ ijmedph.2.4.6 stage renal disease (ESRD), but it is associated with high cardiovascular morbidity \& mortality. In fact, patients with CRF are much more likely to die of cardiovascular complications such as dyslipidemias than to progress to ESRD. ${ }^{2}$

The high coronary heart disease (CHD) prevalence in CRF patients is likely related to their high frequency of dyslipidemia. The characterization of the type of lipid and lipoprotein abnormalities should therefore be considered important in the management of CRF patients to prevent CHD. It has also been suggested that dyslipidemia may contribute to accelerate development of renal insufficiency. ${ }^{3}$ 
The characteristic lipid abnormalities seen in CRF patients are elevated triglycerides, normal/reduced total cholesterol, decreased High Density Lipoprotein (HDL), normal Low Density Lipoprotein (LDL) and raised Lipoprotein(a) [Lp(a)] level. This disturbed lipid pattern accelerates the process of atherosclerosis and impairs the blood supply, further damaging the kidneys. While this process in heart and brain causes morbidity due to cardiovascular and cerebrovascular accidents. ${ }^{4}$

$\mathrm{Lp}(\mathrm{a})$ is a cholesterol-rich lipoprotein with structural similarities to LDL but contains apo(a), a glycoprotein with sequence homology to plasminogen. Lp(a) plays a causal role in the development of atherosclerosis and is a potentially modifiable cardiovascular disease risk factor in hemodialysed CRF patients. ${ }^{5}$ Cardiovascular disease constitutes the major cause of death in patients with ESRD and it is still higher in dialysis patients than in post transplantation patients due to abnormalities in lipids and lipoproteins structure and metabolism. ${ }^{6}$

Controversies exist regarding $L p(a)$ levels in CRF patients with and without hemodialysis. There are few studies that showed $\mathrm{Lp}(\mathrm{a})$ levels were elevated in CRF patients, ${ }^{7,8,9}$ $\mathrm{Lp}(\mathrm{a})$ levels were elevated in hemodialysed patients, ${ }^{10} \mathrm{Lp}(\mathrm{a})$ levels were decreased after hemodialysis ${ }^{8,11}$ and $\mathrm{Lp}$ (a) levels did not vary before and after hemodialysis. ${ }^{5,12}$ With the implication of plasma lipids and lipoproteins in the pathogenesis of atherosclerosis and ischemic heart disease, it becomes worthwhile to study the behavior of various lipid fractions in CRF patients. ${ }^{13}$ Thus the present study was undertaken to compare the levels of $\mathrm{Lp}(\mathrm{a})$ and lipid profile in CRF patients with and without hemodialysis.

\section{METHODOLOGY}

This prospective, observational study was started after prior approval from Institutional Review Board (Human Ethics Committee), Govt. Medical College and Sir T. General Hospital, Bhavnagar, Gujarat (India). Informed consent was obtained from the patients before enrollment. Age and sex matched (Both male and female patients aged 18-60 years) 60 patients of CRF and 30 healthy controls were recruited for this study. They were divide into Group-I (healthy controls), Group-II (CRF patients who never undergone hemodialysis) and Group-III (CRF patients on hemodialysis for more than 6 months). Exclusion criteria include Body mass index (BMI) more than $24.9 \mathrm{~kg} / \mathrm{m}^{2}$, known case of acute renal failure/diabetes mellitus/hypertension/ischemic heart disease, taking drugs that affect lipids and lipoproteins level. $5 \mathrm{ml}$ of venous blood samples were collected in plain tubes after an overnight fast. After collection, the samples were allowed to clot for half an hour following which the samples were centrifuged and serum was analysed. Serum total cholesterol (TC), triglycerides (TGs), HDL cholesterol (HDL-C), LDL cholesterol (LDL-C), Lp(a), urea and creatinine, were measured using commercially available kits in Miura- fully auto analyzer. VLDL cholesterol concentration was calculated using Friedewald's Formula. ${ }^{14}$ Analysis was carried out in Graph Pad Prism 5 project software. All the values obtained were expressed as median with interquartile range. 'Kruskal-Wallis Test' was applied followed by 'Dunn's Multiple Comparison Test' to compare the difference in the medians between controls and study group. Comparison of lipid profile and $\mathrm{Lp}(\mathrm{a})$ levels between male and female patients was carried out in all the three groups by using 'Mann Whitney U Test'. The differences were considered as significant if $\mathrm{p}$ value was $<0.05$.

\section{RESULTS}

A prospective study was taken to determine lipid profile along with $\operatorname{Lp}(\mathrm{a})$ level. The baseline characteristics of subjects are shown in Table 1.

Among 3 groups, 90\% patients of CRF with hemodialysis have elevated triglyceride level and $80 \%$ patients without hemodialysis have elevated triglyceride level as compared to controls $(\mathrm{p}<0.01)$. There is no significant difference observed between total cholesterol levels in all the three groups $(\mathrm{p}>0.05)$.

\begin{tabular}{lccc} 
Table 1: Baseline characteristics of study population & \\
\hline & Group-I Healthy Controls & Group-II CRF pts without HD & Group-III CRF pts with HD \\
\hline No. of participants & 30 & 30 & 30 \\
Age (years) & & & \\
$\quad$ Mean \pm SD & $44.27 \pm 10.72$ & $44.27 \pm 10.72$ & $44.27 \pm 10.72$ \\
$\quad$ Range & $18-60$ & $18-60$ & $18-60$ \\
Sex (male/female) & $18 / 12$ & $18 / 12$ & $18 / 12$ \\
Body mass index $\left(\mathrm{kg} / \mathrm{m}^{2}\right)$ & $22.01 \pm 1.32$ & $21.23 \pm 0.86$ & $20.89 \pm 1.14$ \\
Urea $(\mathrm{mg} / \mathrm{dl})$ & $27.8 \pm 6.58$ & $132.73 \pm 52.55$ & $107.26 \pm 35.27$ \\
Creatinine $(\mathrm{mg} / \mathrm{dl})$ & $0.84 \pm 0.35$ & $11.48 \pm 3.19$ & $6.19 \pm 2.89$ \\
\hline
\end{tabular}

$\mathrm{CRF}=$ chronic renal failure, pts = patients, $\mathrm{HD}=$ hemodialysis. 
HDL cholesterol levels reduced in CRF patients with and without hemodialysis as compared to controls $(<0.05)$. Reduction in HDL cholesterol is observed in $60 \%$ of the CRF patients with hemodialysis as compared to $10 \%$ in CRF patients without hemodialysis $(\mathrm{p}<0.01)$.

There is no significant difference observed between LDL cholesterol levels in all the three groups $(p>0.05)$. VLDL cholesterol levels found to be higher in CRF patients with and without hemodialysis as compared to the controls $(p<0.01)$.

$\mathrm{Lp}$ (a) levels found to be higher in CRF patients with and without hemodialysis as compared to controls $(\mathrm{p}<0.01)$. Elevated Lp(a) levels were observed in $97 \%$ of the CRF patients without hemodialysis and in $90 \%$ of the CRF patients with hemodialysis but the difference was found insignificant $(\mathrm{p}>0.05)$. The biochemical parameters among control and CRF patients with and without hemodialysis has been shown in Table 2.

Out of 30, there were 18 male patients and 12 female patients in each group. Comparison of lipid profile and Lp(a) levels between male and female patients in each group was also carried out to see any effect of gender on these parameters. There was no significant difference observed between lipid profile and Lp(a) levels in male and female patients in CRF patients with and without hemodialysis and in control group ( $p>0.05)$.

\section{DISCUSSION}

In the present study we found hypertriglyceridemia in CRF patients with and without hemodialysis and the levels are even higher in hemodialysed patients as compared to controls. Several studies also showed that the principal lipid abnormality in CRF patients is hypertriglyceridemia. ${ }^{13,15}$ This elevated triglyceride level is because of decreased activity of lipoprotein lipase (LPL) which hydrolyses triglycerides and also enhanced triglyceride synthesis in liver from free fatty acids released from fatty tissue and muscles. ${ }^{10}$
As reported in some of the Indian studies, there is no evidence of hypercholesteremia in earlier stages of CRF but in ESRD cholesterol level is either normal or reduced whereas hypercholesteremia have been reported in some of the western studies. In the present study, we did not find hypercholesteremia instead the levels remain normal or minimally decreased in CRF patients with and without hemodialysis as compared to controls. The values attained were not statistically significant.

CRF results in profound dysregulation of several key enzymes and receptors involved in the metabolism of lipoproteins, particularly those of HDL and triglyceriderich lipoproteins. Downregulation of LCAT, apoA-1, and hepatic lipase together with upregulation of cholesterol ester transfer protein (CETP) are largely responsible for the reduction in HDL cholesterol and elevation of HDL triglyceride in CRF patients with and without hemodialysis. Downregulation of skeletal muscle and adipose tissue LPL, hepatic lipase, and the VLDL receptor and of hepatic LDL Receptor-Related Protein (LRP) is collectively responsible for hypertriglyceridemia, impaired clearance, and elevated plasma levels of VLDL, IDL, and chylomicron remnants despite downregulation of hepatic triglyceride synthetic capacity (DGAT). ${ }^{10,16}$ In our study we found elevated levels of VLDL cholesterol and decreased levels of HDL cholesterol in CRF patients with and without hemodialysis as compared to controls. The difference attained was statistically significant. Amin et al. ${ }^{4}$ reported the same findings.

The level of LDL cholesterol is normal or slightly reduced in CRF patients but there is disturbance in the density distribution of this lipoprotein characterized by the raised level of small, dense particles susceptible to oxidation i.e. oxidized LDL. ${ }^{17}$ In the present study, we did not find raised levels of LDL cholesterol instead the levels remain normal or even decreased in CRF patients with and without hemodialysis as compared to controls. The values attained were not statistically significant. The similar results were forwarded by Saland et al. ${ }^{18}$

\begin{tabular}{lccc}
\multicolumn{4}{l}{ Table 2: The Biochemical parameters among control and CRF patients with and without hemodialysis } \\
\hline Parameters (mg/dl) & Group-I Healthy Controls ( $\mathbf{n = 3 0})$ & Group-II CRF pts without HD (n=30) & Group-III CRF pts with HD (n=30) \\
\hline TC & $169.0(162.3,184.8)$ & $180.3(137.5,197.8)$ & $169.0(157.5,178.0)$ \\
TGs & $135.5(125.0,155.3)$ & $209.0^{* *}(200.0,216.0)$ & $240.5^{\star *}(206.0,272.0)$ \\
HDL-C & $45.00(35.00,54.25)$ & $38.16^{* *}(30.75,53.25)$ & $28.00^{* *}(24.75,31.05)$ \\
LDL-C & $103.5(97.00,114.3)$ & $102.0(88.00,116.3)$ & $102.5(97.50,111.0)$ \\
VLDL-C & $27.10(24.75,31.05)$ & $42.00^{* *}(40.00,43.25)$ & $48.10^{* *}(41.20,54.40)$ \\
Lp(a) & $10.00(5.00,11.25)$ & $38.00^{* *}(32.75,43.25)$ & $38.50^{* *}(32.00,48.50)$ \\
\hline
\end{tabular}

$* p<0.05 * * p<0.01$. Figures in parentheses indicate the number of patients. $C R F=$ chronic renal failure, $p t s=$ patients, $H D=$ hemodialysis 
$\mathrm{Lp}(\mathrm{a})$ is LDL-like particle formed by the association of the highly polymorphic glycosylated apo (a) with apo B100, the classic protein moiety of LDL. ${ }^{19}$ The kidney may be involved in the turnover of $\operatorname{Lp}(\mathrm{a})$, and it is suggested that renal failure interferes with normal catabolism of $\operatorname{Lp}(a) .{ }^{8} \mathrm{Lp}(\mathrm{a})$ shows both atherogenic and thrombogenic properties by various mechanisms that play a causal role in atherosclerosis. ${ }^{20} \mathrm{In}$ CRF, besides other lipid abnormalities, there is significant increase in the level of serum $\mathrm{Lp}(\mathrm{a}){ }^{8}$ Dialysis therapy may increases $\mathrm{Lp}(\mathrm{a})^{10}$ or decreases $\mathrm{Lp}$ (a) level..$^{8,11}$ Some studies also reported that Lp(a) level did not vary before and after hemodialysis. ${ }^{5,12}$ In our study we find elevated levels of $\mathrm{Lp}(\mathrm{a})$ in CRF patients with and without hemodialysis as compared to controls. The difference was found highly significant. When we compared the levels of $\mathrm{Lp}$ (a) between group-II and group-III, the difference was found insignificant. We also compared the levels of lipid profile and $\operatorname{Lp}(\mathrm{a})$ between male and female patients in all the three groups. We found that both male and female patients were equally affected $(p>0.05)$. The loss of renal function in hemodialysed patients causes elevated $\operatorname{Lp}(\mathrm{a})$ plasma levels because of decreased clearance but not increased production of $\operatorname{Lp}(\mathrm{a})$. The prolonged retention time of $\mathrm{Lp}(\mathrm{a})$ in hemodialysed patients might importantly contribute to the high risk of atherosclerosis in these patients. ${ }^{21}$

\section{CONCLUSION}

CRF patients with and without hemodialysis are at greater risk of development of dyslipidemias, characterized by hypertriglyceridemia, elevated VLDL-C and Lp(a) levels and decreased HDL-C levels. Total cholesterol and LDL cholesterol levels remain normal or decreased in these patients. Both male and female patients of CRF with and without hemodialysis have dyslipidemias without any discrimination of sex and it is not attenuated by the hemodialysis process. These lipid and lipoprotein abnormalities together enhance the risk of developing cardiovascular disease in these patients. A strict monitoring of lipid profile and lipoproteins particularly $\mathrm{Lp}$ (a) can reduce the morbidity and mortality rate and will also improve the quality of life of CRF patients.

\section{CONFLICT OF INTEREST NOTIFICATION}

None

\section{REFERENCES}

1. William JM, Stephen KB, editors. Clinical Biochemistry: metabolic and clinical aspects. $2^{\text {nd }}$ ed. Philadelphia: Churchill Livingstone, Elsevier; 2008: pp. 145, 154.

2. Godela B, Mony F. CME topic on Chronic Kidney Disease: Whom to Screen and How to Treat, Part 1: Definition, Epidemiology and Laboratory Testing. Southern Medical Journal 2010; 103(2):140-6.

3. Victor B. Dyslipidemia at CRF. eJIFCC 2009; 20(1): 58-65.

4. Amin K, Javed M, et al. Abid M. Pattern of dyslipidemia in patients with CRF. Prof Med J 2006; 13(1): 79-84.

5. Cressman MD, Heyka RJ, Paganini EP, et al. Lipoprotein(a) is an independent risk factor for cardiovascular disease in hemodialysis patients. Circulation 1992; 86(2): 475-82.

6. Fauci AS, Kasper DL, Longo DL, Braunwald E, Hauser SL, Larry Jameson J, Loscalzo J, editors. Harrison's principles of Internal Medicine. $17^{\text {th }}$ ed. New York: McGraw Hill, Health Professions Division 2008: pp. 1772-6.

7. Kronenberg F, Lingenhel A, Neyer U, et al. Prevalence of Dyslipidemic risk factors in hemodialysis and CAPD patients. Kidney International 2003; 63(Suppl 84): 113-16.

8. Kalra OP, Khaira A, Gambhir JK, et al. Lipoprotein(a) in Chronic Renal Failure: Effect of Maintenance Hemodialysis. Hemodial Int 2003; 7(4): 326-33.

9. Yerkey MW, Kernis SJ, Franklin BA, et al. Renal Dysfunction and Acceleration of Coronary Disease. Heart 2004; 90: 961-6.

10. Janicki K, Molas G, Furmaga J, et al. Abnormal lipoprotein metabolism in hemodialysis patients, Annales Universitatis Mariae Curie - Sklodowska Lublin Polonia 2007; 62: 311-14.

11. Kandoussi A, Cachera C, Pagniez D, et al. Plasma level of lipoprotein $\mathrm{Lp}(\mathrm{a})$ is high in predialysis or hemodialysis, but not in CAPD. Kidney International, 1992; 42: 424-5.

12. Barbagallo CM, Averna MR, Sparachio $V$, et al. Lp (a) levels in end-stage renal failure and renal transplantation. Nephron 1993; 64(abstract): 560-4.

13. Ravichandran RR, Nerurkar SV, Acharya VN, et al. Hyperlipidemia in patients with chronic renal failure. Journal of Post Graduate Medicine 1983; 29(4): 212-17.

14. Friedewald WT, Levy RI, Fredrickson DS. Estimation of the concentration of low-density lipoprotein cholesterol in plasma without the use of the preparative ultracentrifuge. Clin Chem 1992; 18: 499-502.

15. Shah B, Nair S, Sirsat RA, et al. Dyslipidemia in Patients of CRF and in renal transplant patients. J post Grad Med 1994; 40(2): 57-60.

16. Vaziri ND. Dyslipidemia of chronic renal failure: the nature, mechanisms, and potential consequences. Am J Physiol Renal Physiol 2006; 290(2): F262-72.

17. Majumdar A, Wheeler DC. Lipid abnormalities in renal disease. J R Soc Med 2000; 93:178-82.

18. Saland JM, Ginsberg HN. Lipoprotein metabolism in chronic renal insufficiency. Pediatr Nephrol 2007; 22:1095-1112

19. Lippi G, Guidi G. Lipoprotein(a): from ancestral benefit to modern pathogen?, Q J Med 2000; 93:75-84.

20. Deb A, Caplice NM. Lipoprotein(a): New Insights into Mechanisms of Atherogenesis and Thrombosis. Clin. Cardiol. 2004; 27: 258-64.

21. Frischmann ME, Kronenberg F, Trenkwalder E, et al. In vivo turnover study demonstrates diminished clearance of lipoprotein(a) in hemodialysis patients. Kidney International, 2007; 71(abstract): 1036-43. 